\title{
ANALOG PHASE MODULATION FOR AVIONICS APPLICATIONS
}

\author{
Vincent J. Urick, Frank Bucholtz, Preetpaul S. Devgan, and Jason D. McKinney \\ U.S. Naval Research Laboratory, Photonics Technology Branch \\ Washington, DC 20375
}

\section{Introduction}

Radio-frequency (RF) photonics and fiber optics will play an important role in future airborne applications, leveraging advantages over traditional RF cabling such as decreased size and weight, increased flexibility, lower signal loss in fiber, larger bandwidth, and invulnerability to electromagnetic interference. In military electromagnetic warfare applications, RF links can be used route microwave signals throughout the airframe where analog-to-digital conversion is not practical or possible. Traditionally, intensity modulation with direct detection architectures have been proposed for such links, but here we suggest phase modulation with interferometric detection as an alternative.

Phase modulation with interferometric detection [1] offers numerous advantages over intensity modulation with direct detection. For example, a phase modulator requires no electronics as opposed to a Mach-Zehnder modulator (MZM), which requires a quadrature bias voltage. This is particularly advantageous in airborne applications where the modulator may be required in a harsh environment such as on the wingtip or in a sensor pod. In addition, the ruggedization of a phase modulator should be more practical than that for the multi-path MZM architecture, owing to a phase modulator's relative simplicity. We will show below that phase modulation with interferometric detection can outperform intensity modulation with direct detection in terms of all RF performance metrics. While not of immediate concern in avionic applications, where the transmission lengths do not exceed a few hundred meters, the constant-intensity phase modulation format is also less susceptible to fiber nonlinearities. The tradeoffs for the benefits afforded by phase modulation with interferometric detection are reduced bandwidth and increased complexity at the terminal end of the RF link.

\section{Analog Phase Modulation Performance Metrics}

The architecture for a phase-modulated analog link employing interferometric detection is shown in Figure 1, along with a measured response for such a link. The architecture comprises a laser, a phase modulator $(\Phi M)$, the fiber-optic cabling, and an asymmetric Mach-Zehnder interferometer (MZI) with balanced photodiodes (PDs) on its output. The differential time delay in the MZI $(\tau)$ dictates the frequency response for these links, along with the modulator half-wave voltage $\left(V_{\pi}\right)$ and DC photocurrent per detector $\left(I_{\mathrm{dc}}\right)$. For the data in Figure $1, \tau=400 \mathrm{ps}, V_{\pi}=6.9 \mathrm{~V}$ at $5 \mathrm{GHz}$, and $I_{\mathrm{dc}}=5 \mathrm{~mA}$.

We will not derive the RF performance for a phase-modulated link with interferometric detection here. Rather, we state that the performance is governed by the following equations:
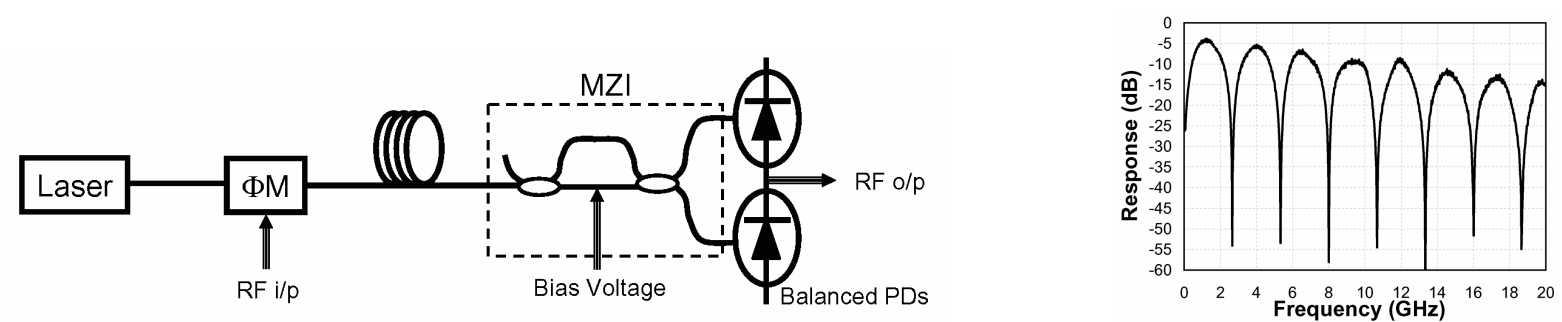

Figure 1. The architecture for a phase-modulated link employing balanced interferometric detection and a measured response for a link with a differential delay of approximately $400 \mathrm{ps}$. 
Table 1. Comparison Between Phase- and Intensity-Modulated Analog Links in the Shot-Noise Limit

\begin{tabular}{|l|c|c|c|}
\hline & IMDD $@ I_{\mathrm{dc}}$ & $\begin{array}{c}\boldsymbol{\Phi M I D} @ I_{\mathrm{dc}} \\
\text { per Diode }\end{array}$ & $\begin{array}{c}\boldsymbol{\Phi M I D ~} @ I_{\mathrm{dc}} / \mathbf{2} \\
\text { per Diode }\end{array}$ \\
\hline RF Gain & $G_{\mathrm{IMDD}}=\left(\frac{I_{\mathrm{dc}}}{V_{\pi}}\right)^{2} \pi^{2} Z_{\mathrm{in}} Z_{\mathrm{out}}$ & $G_{\mathrm{IMDD}}+12.0 \mathrm{~dB}$ & $G_{\mathrm{IMDD}}+6.0 \mathrm{~dB}$ \\
\hline RF Noise Figure & $N F_{\mathrm{IMDD}}=\frac{2 e V_{\pi}^{2}}{I_{\mathrm{dc}} \pi^{2} k T Z_{\mathrm{in}}}$ & $N F_{\mathrm{IMDD}}-9.0 \mathrm{~dB}$ & $N F_{\mathrm{IMDD}}-6.0 \mathrm{~dB}$ \\
\hline $\begin{array}{l}\text { Compression Dynamic } \\
\text { Range }\end{array}$ & $C D R_{\mathrm{MMD}}=\frac{I_{\mathrm{dc}} \phi_{1 \mathrm{~dB}}^{2}}{4 e}$ & $C D R_{\mathrm{IMDD}}+4.3 \mathrm{~dB}$ & $C D R_{\mathrm{MMD}}+1.3 \mathrm{~dB}$ \\
\hline $\begin{array}{l}\text { Spurious-Free Dynamic } \\
\text { Range }\end{array}$ & $S F D R_{\mathrm{IMDD}}=\left(\frac{2 I_{\mathrm{dc}}}{e}\right)^{2 / 3}$ & $S F D R_{\mathrm{MMDD}}+2.0 \mathrm{~dB}$ & $S F D R_{\mathrm{IMDD}}$ \\
\hline
\end{tabular}

$$
\begin{array}{cc}
G_{\mathrm{rf}}=16\left(\frac{I_{\mathrm{dc}}}{V_{\pi}}\right)^{2} \pi^{2} Z_{\text {in }} Z_{\text {out }} \sin ^{2}\left(\frac{\Omega \tau}{2}\right) & N F_{\text {rf }}=\frac{R I N_{\text {total }} V_{\pi}^{2}}{4 \pi^{2} k T Z_{\text {in }} \sin ^{2}(\Omega \tau / 2)} \\
C D R=\frac{2 \phi_{1 \mathrm{~dB}}^{2} \sin ^{2}(\Omega \tau / 2)}{R I N_{\text {total }}} & S F D R_{\text {max }}=\left(\frac{4}{R I N_{\text {total }}}\right)^{2 / 3} .
\end{array}
$$

Here, $G_{\mathrm{rf}}$ is the RF gain, $N F_{\text {rf }}$ is the RF noise figure, $C D R$ is the compression dynamic range, and $S F D R_{\max }$ is the spurious-free dynamic range at the peak response; $Z_{\text {in }}$ is the input impedance, $Z_{\text {out }}$ is the output impedance, $\Omega$ is the angular radio frequency, $k$ is Boltzmann's constant, $T$ is the temperature, $\phi_{1 \mathrm{~dB}}$ is the phase-shift amplitude at $1-\mathrm{dB}$ compression, and $R I N_{\text {total }}$ is the total output-referenced relative intensity noise. Generally, $R I N_{\text {total }}$ will contain contributions from thermal noise, optical amplifier noise, laser noise and shot noise. The periodic nature of the RF gain shown in Figure 1 should be apparent in the functional dependence of $G_{\mathrm{rf}}$ on $\Omega$. It follows that the total RF performance, as dictated by the four metrics above, is optimized at peak response, where $\Omega \equiv x \pi / \tau$ with $x$ being an odd integer. We strongly note that the response shown in Figure 1 is for a specific value of $\tau$, and that almost any frequency can be covered by properly choosing $\tau$.

To compare the performance of a phase-modulated analog link with interferometric detection to an intensity-modulated direct-detection link, we list the calculated performance for each in Table 1. The subscripts "IMDD" and "ФMID" in Table 1 correspond to intensity and phase modulation, respectively, and $e$ is the electron charge constant (taken to be positive). In Table 1 we consider shot noise, noting that shot-noise-limited performance can easily be achieved in both architectures. We tabulate the results for phase modulation at peak response and consider two values for the DC photocurrent for a fair comparison. The important result is that phase-modulated analog links at the peak response substantially outperform intensity-modulated analog links.

\section{Conclusion}

We have stated that phase modulation can outperform intensity modulation in terms of RF gain, RF noise figure, compression dynamic range, and spurious-free dynamic range. We cite the additional benefits of modulator simplicity and higher tolerance to fiber nonlinearities for the phase-modulation architecture. Complexity at the terminal end of the RF link and reduced bandwidth due to the interferometric demodulation are the tradeoffs for these benefits. However, as described above, most frequencies can be covered with a properly-chosen demodulating interferometer. Additionally, the complexity at the terminal end of the RF link can be acceptable when the demodulator is remoted to a less-harsh environment such as the cockpit. Therefore, we suggest that phase modulation should be seriously considered for RF photonic links in avionics applications.

\section{References}

[1] M. J. LaGasse and S. Thaniyavarn, "Bias-free high-dynamic-range phase-modulated fiber-optic link," IEEE Photon. Technol. Lett., vol. 9, no. 5, pp. 681-683, May 1997. 